\title{
Atividade antioxidante de pimentas do gênero Capsicum
}

\author{
Antioxidant activities of peppers of the genus Capsicum
}

\section{Luciene Mendonça da COSTA ${ }^{1 *}$, Neusa Fernandes de MOURA ${ }^{1}$, Cristiane MARANGONI ${ }^{1}$, Caroline Eliza MENDES ${ }^{2}$, Alexandre de Oliveira TEIXEIRA ${ }^{3}$}

\section{Resumo}

Foi avaliada a atividade antioxidante pelo Sistema $\beta$-caroteno/Ácido Linoleico, ensaio do radical DPPH• (2,2-difenil-1-picrilhidrazila), do extrato bruto (EB) e frações hexânica (FH), clorofórmica (FC) e acetato de etila (FA) das pimentas malagueta (C. frutescens), cambuci (C. baccatum var. pendulum), cumari (C. baccatum var praetermissum) e pimentão magali (C. annuum var. annuum). As concentrações de capsaicinoides e de fenólicos totais presentes nas pimentas também foram determinadas. Os resultados obtidos demonstraram que as FC e FA das pimentas apresentaram maior concentração de fenólicos totais e capsaicinoides, sendo que a pimenta cumari foi a espécie com maior concentração destes compostos. A melhor atividade antioxidante pelo Sistema $\beta$-caroteno/Ácido Linoleico, foi obtida para o EB e FA da pimenta cambuci, seguida do EB da pimenta malagueta. Pelo método DPPH a FC e FA apresentaram menores valores de EC $_{50}$, sendo a pimenta cumari e cambuci as mais efetivas. Estes resultados demonstram que as pimentas cumari, cambuci e malagueta podem ser utilizadas como agentes antioxidantes naturais em alimentos.

Palavras-chave: Capsicum; pimentas; atividade antioxidante.

\begin{abstract}
The antioxidant activity was evaluated by the $\beta$-Carotene/Linoleic Acid System, DPPH (2,2-diphenyl-1-picrylhydrazyl) radical assay, to the crude extract (EB) and fractions hexanic (FH), chloroformic (FC), and ethyl acetate (FA) of malagueta peppers (C. frutescens), cambuci (C. baccatum var. pendulum), cumari (C. baccatum var praetermissum), and pepper magali (C. annuum var. annuum). The concentration of total phenolics and capsacinoids present in the peppers was also determined. The results showed that the FC and the FA peppers showed higher concentrations of total phenolics and capsacinoids, and that the cumari pepper was the species with higher concentrations of these compounds. The best antioxidant activity according to the $\beta$-Carotene/Linoléico Acid System were obtained for the EB and the FA cambuci pepper, followed by the EB malagueta pepper. According to the DPPH method, the FC and FA showed lower values of EC ${ }_{50}$, and the cumari and cambuci peppers were the most effective. The results show that cumari, cambuci, and malagueta peppers can be used as natural antioxidant agents in food.
\end{abstract}

Keywords: Capsicum; peppers; antioxidant activities.

\section{Introdução}

Naturalmente os alimentos estão em constantes modificações, representadas por alterações físicas, enzimáticas, microbiológicas e químicas, como as reações de oxidação de lipídios e outros nutrientes susceptíveis à ação do oxigênio e radicais livres, provocando por sua vez o aparecimento de sabores e odores desagradáveis, modificações do valor nutricional e a diminuição da vida de prateleira dos alimentos.

Para retardar tais processos, a indústria faz uso de aditivos químicos constantemente questionados pelos consumidores quanto aos seus possíveis efeitos negativos à saúde. Neste contexto, há grande interesse em encontrar nas plantas condimentares, princípios ativos que possuam ação antioxidante e que possam contribuir para a conservação de alimentos, sendo estes produzidos sem danos ao meio ambiente e à saúde humana.

Plantas condimentares, tais como as pimentas e pimentões do gênero Capsicum, que sempre foram usadas pelos índios e civilizações antigas para tornar os alimentos mais agradáveis ao paladar, além de serem utilizadas como conservantes em alimentos, são fontes de antioxidantes naturais como a vitamina $\mathrm{E}$, vitamina $\mathrm{C}$ e carotenoides (REIFSCHNEIDER, 2000). Estas pimentas também são ricas em capsaicinoides, compostos fenólicos responsáveis pelo sabor pungente ou picante (CARVALHO; BIACHETTI, 2004).

O Brasil é o segundo maior produtor de pimenta no mundo (RISTORI et al., 2002) e centro da diversidade do gênero Capsicum (REIFSCHNEIDER, 2000). Essa hortaliça está difundida em todas as regiões do Brasil, sendo que as principais áreas de cultivo são as regiões Sudeste e Centro-Oeste. São comercializadas para o consumo in natura, conservas caseiras e exportação do produto industrializado (WAGNER, 2003).

O presente trabalho teve como objetivo avaliar as atividades antioxidante do extrato bruto e frações hexânica, clorofórmica e

Recebido para publicação em 31/1/2008

Aceito para publicação em 3/1/2009 (003199)

${ }^{1}$ Ciências Ambientais, Universidade Comunitária Regional de Chapecó - Unochapecó, Chapecó - SC, Brasil, E-mail: luciene.mc@gmail.com

${ }^{2}$ Centro de Ciências Agroambientais e Alimentos, Curso de Engenharia Química, Universidade Comunitária Regional de Chapecó - Unochapecó, Chapecó - SC, Brasil

3 Departamento de Engenharia de Biossistemas, Universidade Federal de São João Del-Rei - UFSJ, São João Del-Rei - MG, Brasil

${ }^{*}$ A quem a correspondência deve ser enviada 
acetato de etila, das pimentas malagueta (C. frutescens), cambuci (C. baccatum var. pendulum), pimenta cumari (C. baccatum var. praetermissum) e pimentão magali (C. annuum var. annuum).

\section{Material e métodos}

\subsection{Material}

Foram utilizados os frutos maduros da pimenta malagueta (C. frutescens) cambuci (C. baccatum var. pendulum), pimenta cumari (C. baccatum var. praetermissum) e pimentão magali (C. annuum var. annuum) coletados em Chapecó/SC.

Para obtenção do extrato Bruto (EB), os frutos com sementes foram lavados sob água corrente e picados em pedaços pequenos, seguido de maceração em solução hidroalcoólica (Etanol $96^{\circ} \mathrm{GL}$ ). Posteriormente o EB sofreu extração líquidolíquido pelo sistema de solventes com grau de polaridade crescente, resultando em Fração Hexânico (FH), Fração Clorofórmico (FC) e Fração Acetato de Etila (FA).

\subsection{Métodos}

\section{Quantificação de capsaicinoides}

Para determinar o teor de Capsaicinoides das pimentas Capsicum, foi utilizada a metodologia descrita por Sadasivam e Manikkam (1992), na qual a concentração é estimada pela medida espectrofotométrica da cor azul formada pela redução do ácido fosfomolibídico a ácido fosfomolibidênico.

Pesou-se 0,05 g dos extratos brutos e fracionados das pimentas estudadas, que foram diluídas em $100 \mathrm{~mL}$ de água destilada, retirou-se $1 \mathrm{~mL}$ desta solução que foi misturada em $5 \mathrm{~mL}$ de $\mathrm{NaOH}$ a $0,4 \%$ e $3 \mathrm{~mL}$ de ácido fosfomolibídico a $3 \%$. A mistura foi deixada em repouso ao abrigo de luz por 1 hora. Após o período de reação, as absorbâncias das amostras foram lidas através do espectrofotômetro de UV visível (marca SCINCOSUV, modelo 2120), no comprimento de onda de $650 \mathrm{~nm}$.

A curva foi construída misturando-se a solução de Capsaicinoides a 100\% (65\% de Capsaicina, 20\% de Dihidrocapsaicina e $15 \%$ de Homodihidrocapsaicina) em diferentes concentrações em $5 \mathrm{~mL}$ de $\mathrm{NaOH}$ a $0,4 \%, 3 \mathrm{~mL}$ de ácido fosfomolibídico a 3\%. Os resultados foram expressos em $\mathrm{mg}$ de Capsaicinoides por $100 \mathrm{~g}$ de amostra.

\section{Quantificação de fenólicos totais}

Para quantificação dos fenólicos totais utilizou-se o reagente Folin-Denis descrito pelo método 9110 da AOAC (1980).

Preparou-se inicialmente a solução supersaturada de carbonato de sódio e o reagente Folin-Denis. A mistura foi fervida em refluxo durante duas horas e após o resfriamento diluído em água 1:1, sendo acondicionada em recipiente protegido da luz.

Para preparação da curva de calibração do catecol, foram pipetadas diferentes alíquotas da solução padrão de catecol em balóes volumétricos de $100 \mathrm{~mL}$ contendo $5 \mathrm{~mL}$ de reagente de Folin-Denis e $10 \mathrm{~mL}$ da solução de carbonato de sódio. As concentrações foram homogeneizadas em Vortex e deixadas em repouso ao abrigo da luz por 30 minutos, para então serem realizadas as leituras em espectrofotômetro de UV visível (marca SCINCOSUV, modelo 2120) a $760 \mathrm{~nm}$.

Os ensaios foram realizados diluindo-se $0,05 \mathrm{~g}$ de extratos e frações das pimentas, em $100 \mathrm{~mL}$ de água destilada em balão volumétrico. Transferiu-se $1 \mathrm{~mL}$ desta solução para outro balão de $100 \mathrm{~mL}$ contendo $5 \mathrm{~mL}$ de reagente Folin-Denis, $10 \mathrm{~mL}$ de solução supersaturada de carbonato de sódio e avolumou-se com água. Foram mantidas em repouso e ao abrigo da luz por 30 minutos. Preparou-se o branco para o controle negativo.

A quantidade de fenólicos totais foi obtida através da seguinte equação: $\mathrm{FT}=$ leitura $(\mathrm{mg} / \mathrm{mL}) \times 100 /$ peso da amostra $(\mathrm{g})$. Onde: Leitura é a concentração de catecol obtida na curva de calibração de catecol referente à absorbância lida para a amostra.

\section{Avaliação da atividade antioxidante pelo sistema $\beta$-caroteno/ ácido linoleico}

A atividade antioxidante foi determinada pelo sistema $\beta$-caroteno/ácido linoleico seguindo a metodologia descrita por Marco (1968).

Para a preparação da emulsão $\beta$-caroteno/ácido linoleico, adicionou-se $1 \mathrm{mg}$ de $\beta$-caroteno em $10 \mathrm{~mL}$ de clorofórmio, homogeneizou-se e transferiu-se $1 \mathrm{~mL}$ desta solução para um balão de fundo redondo contendo $25 \mu \mathrm{L}$ de ácido linoleico e $200 \mu \mathrm{L}$ de Tween 40 . O clorofórmio da mistura foi evaporado em rota evaporador a $40^{\circ} \mathrm{C}$, por 10 minutos. Após a evaporação, foi adicionada a mistura $50 \mathrm{~mL}$ de água destilada aerada (saturada em oxigênio por 30 minutos) e, em seguida, agitada para formar emulsão.

Foram retirados $5 \mathrm{~mL}$ da emulsão de $\beta$-caroteno/ácido linoleico para a leitura do branco; para o controle positivo misturaram-se $5 \mathrm{~mL}$ da emulsão com $0,2 \mathrm{~mL}$ de BHT e pipetaramse $5 \mathrm{~mL}$ da emulsão para adição de $1 \mathrm{mg}$ de extrato bruto e frações hexânica, clorofórmica e acetato de etila das quatro espécies de pimentas. Em seguida, foram aquecidas em banho-maria a $50^{\circ} \mathrm{C}$ por 15 minutos e depois esfriadas por 30 minutos. A leitura foi realizada em espectrofotômetro com comprimento de onda de $470 \mathrm{~nm}$ em intervalos de 15 minutos por 2 horas. A atividade foi determinada em porcentagem (\%) de Atividade Antioxidante (AA) conforme equação descrita abaixo: AA(\%) = 100 - (Absorbância inicial - Absorbância final) Amostra × 100/Absorbância inicial - Absorbância final) Branco.

\section{Avaliação da atividade antioxidante pelo método DPPH (2,2-difenil-1-picrilhidrazila)}

O método utilizado para a determinação da atividade antioxidante via radical DPPH (2,2-difenil-1-picrilhidrazila) foi desenvolvido por Brand-Willians, Cuvelier e Berset (1995) com modificações por Mensor et al. (2001).

Inicialmente foi preparada a solução estoque de DPPH, na qual $2 \mathrm{mg}$ de DPPH foram solubilizados em $50 \mathrm{~mL}$ de álcool metílico 96\%. Para a solução das amostras, pesaram-se $40 \mathrm{mg}$ dos extratos e frações de cada pimenta estudada, que foram misturadas em $10 \mathrm{~mL}$ de álcool metílico $96^{\circ} \mathrm{GL}$. 
Em tubos contendo $1 \mathrm{~mL}$ da solução estoque de DPPH, adicionaram-se álcool metílico $96 \%$ e a solução das amostras, obtendo-se as concentrações de 5, 10, 25, 50, 125 e $250 \mu \mathrm{g} \cdot \mathrm{mL}^{-1}$ de amostra. As amostras contidas nos tubos foram homogeneizadas e deixadas em repouso ao abrigo de luz por 30 minutos. Um controle negativo foi feito com 1,5 mL de metanol $\left(96^{\circ} \mathrm{GL}\right)$ e $1 \mathrm{~mL}$ de solução DPPH. Para o controle positivo pipetou-se $0,8 \mathrm{~mL}$ de BHT que foi misturado a $1 \mathrm{~mL}$ da solução estoque de DPPH e a 0,7 mL de álcool metílico 96\%.

Após 30 minutos, a absorbância foi lida em $517 \mathrm{~nm}$ em espectrofotômetro de UV visível (marca SCINCOSUV, modelo 2120) e foi convertida em percentagem de atividade antioxidante (AA\%) utilizando-se a seguinte equação: AA (\%) = $100-$ (Absorbância da amostra) × 100/(Absorbância controle). Os dados do método DPPH também podem ser interpretados pelo $\mathrm{CE}_{50}$, ou seja, a concentração da amostra em que $50 \%$ da atividade antioxidante (antirradical) é observada.

\section{Análise estatística}

As análises foram realizadas em duplicata com três repetições. Os dados foram submetidos à análise de variância (teste $F$ ) e as médias comparadas pelo Teste de Tukey (5\%) e Dunnett (5\%) através do programa Estatistic 6.0.

\section{Resultados e discussão}

\subsection{Quantificação de capsaicinoides}

As concentrações de capsaicinoides encontrados para o extrato bruto e fracionado das espécies C. frutescens (malagueta), C. annuum var. annuum (pimentão magali), C. baccatun var. pendulum (cambuci), C. baccatun var. praetermissum (cumari) estão apresentadas na Tabela 1 .

Os resultados obtidos para a pimenta malagueta demonstram que a fração clorofórmica apresenta maior concentração $(\mathrm{p}<0,05)$ de capsaicinoides seguida da FA, enquanto que o extrato bruto e fração hexânica foi o de menor concentração $(\mathrm{p}<0,05)$. Este resultado foi observado também para a pimenta cumari.

O pimentão magali foi a espécie que apresentou menor concentração de capsaicinoides, com o extrato e suas frações não apresentaram diferença entre si.

Entre todos os extratos e frações analisados, a fração clorofórmica da pimenta cumari apresentou maior concentração de capsaicinoides. Pimenta esta extremamente pungente.

As frações clorofórmica e acetato de etila da pimenta cambuci apresentaram maior concentração de capsaicinoides em relação ao EB e FH, não deferindo entre si.

Observou-se que as maiores concentrações foram encontradas nas frações clorofórmica e acetato de etila e menores no extrato bruto e fração hexânica. Tais resultados podem ser explicados pelo fato de solventes mais polares (BERTOLDI, 2006) extraírem normalmente maior quantidade de fenólicos, e os capsaicinoides fazem parte desta classe de compostos.

Das dez variedades de extratos brutos de pimentas C. annuum pesquisadas por Deepa et al. (2007), foram encontradas concentrações de capsaicina variando de 776-1440 $\mu \mathrm{g} .100 \mathrm{~g}^{-1}$ para pimentas imaturas (verdes) e $277-1529 \mu \mathrm{g} .100 \mathrm{~g}^{-1}$ para pimentas maduras (vermelhas). Resultados inferiores aos determinados no presente trabalho.

Tabela 1. Concentração de capsaicinóides $\left(\mathrm{mg} \cdot 100 \mathrm{~g}^{-1}\right)$ no extrato bruto e fracionado de pimentas Capsicum.

\begin{tabular}{|c|c|c|}
\hline Pimenta & Extrato e frações & Capsaicinóides (mg.100 g $\left.\mathrm{g}^{-1}\right)$ \\
\hline \multirow[t]{4}{*}{ C. frutescens (malagueta) } & Bruto & $9,22 \pm 0,35^{\mathrm{e}}$ \\
\hline & Hexânico & $4,76 \pm 0,83^{e}$ \\
\hline & Clorofórmico & $235,80 \pm 8,16^{c}$ \\
\hline & Acetato de etila & $125,11 \pm 12,74^{\mathrm{d}}$ \\
\hline Média & & $93,72^{\mathrm{C}}$ \\
\hline \multirow[t]{4}{*}{ C. annuum var. annuum (pimentão magali) } & Bruto & $3,52 \pm 0,41^{\mathrm{e}}$ \\
\hline & Hexânico & $10,58 \pm 0,10^{\mathrm{e}}$ \\
\hline & Clorofórmico & $11,52 \pm 0,18^{\mathrm{e}}$ \\
\hline & Acetato de etila & $11,58 \pm 0,37^{\mathrm{e}}$ \\
\hline Média & & $9,30^{\mathrm{D}}$ \\
\hline \multirow[t]{4}{*}{ C. baccatun var. pendulum (cambuci) } & Bruto & $6,17 \pm 0,10^{\mathrm{e}}$ \\
\hline & Hexânico & $10,81 \pm 0,53^{\mathrm{e}}$ \\
\hline & Clorofórmico & $234,62 \pm 9,35^{c}$ \\
\hline & Acetato de etila & $248,75 \pm 15,40^{c}$ \\
\hline Média & & $125,09^{\mathrm{B}}$ \\
\hline \multirow[t]{4}{*}{ C.baccatun var. praetermissum (cumari) } & Bruto & $10,58 \pm 0,27^{\mathrm{e}}$ \\
\hline & Hexânico & $9,05 \pm 0,31^{\mathrm{e}}$ \\
\hline & Clorofórmico & $560,80 \pm 16,69^{\mathrm{a}}$ \\
\hline & Acetato de etila & $515,24 \pm 21,49^{b}$ \\
\hline Média & & $274,24^{\mathrm{A}}$ \\
\hline
\end{tabular}

Letras iguais, minúsculas (média dos extratos) e maiúsculas (média das pimentas) na mesma coluna não diferem ( $\mathrm{p}>0,05)$ pelo teste Tukey. 
Materska e Perucka (2005) obtiveram para extrato metanólico de C. annuum L. 0,442 $\mathrm{mg} \cdot \mathrm{g}^{-1}$ de capsaicina e $0,317 \mathrm{mg} \cdot \mathrm{g}^{-1}$ de dihidrocapsaicina para frutos imaturos, para frutos maduros $0,530 \mathrm{mg} \cdot \mathrm{g}^{-1}$ de capsaicina e $0,350 \mathrm{mg} \cdot \mathrm{g}^{-1} \mathrm{de}$ dihidrocapsaicina.

Perucka e Oleszek (2000) determinaram concentrações de capsaicinoides em extrato metanólico de C. annuum L. por meio de espectrofotômetro e CLAE (Cromatografia Líquida de Alta Performance), 0,706 e 0,715 mg.g ${ }^{-1}$, respectivamente. Os autores ainda constataram que a determinação de capsaicinoides por espectrofotometria foi comparável a do CLAE. Já Topuz e Ozdemir (2007) encontraram para cultivares de $C$. annuum a concentração de capsaicinoides que variou de 471,3 a $688,1 \mathrm{mg} / \mathrm{kg}$ na base seca.

Pino et al. (2007) comprovaram a concentração de capsaicinoides com a cor (vermelho, alaranjados e marrons) de 10 pimentas $C$. chinense cultivados em Cuba. Verificaram que o índice de capsaicinoides das pimentas variou de 41,8 a $65,9 \mathrm{mg} \cdot 100 \mathrm{~g} \mathrm{~g}^{-1}$ da fruta seca. Especificamente os cultivares alaranjados foram os mais pungentes com concentração de capsaicinoides de $55 \mathrm{mg} .100 \mathrm{~g}^{-1}$ da fruta seca enquanto os vermelhos $45 \mathrm{mg} \cdot 100 \mathrm{~g}^{-1}$ da fruta seca.

\subsection{Quantificação de fenólicos totais}

A concentração de fenólicos totais, expressos em equivalente de catecol por $100 \mathrm{~g}$ de amostra encontrados para os extratos brutos e frações das espécies C. frutescens (malagueta), C. annuum var. annuum (pimentão magali), C. baccatun var. pendulum (cambuci), C.baccatun var. praetermissum (cumari), está apresentada na Tabela 2.
A pimenta malagueta apresentou maior concentração de fenólicos totais na fração clorofórmica, seguida do acetato de etila. As menores concentrações encontram-se no extrato bruto e fração hexânica.

Ao analisar os resultados das pimentas cambuci e cumari, constata-se que: as maiores concentrações de fenólicos totais foram extraídas pelos solventes clorofórmio e acetato de etila, não havendo diferença entre eles; para os extratos brutos e frações hexânicas, as menores concentrações, não havendo diferença estatística entre os resultados.

O pimentão apresenta concentrações inferiores de compostos fenólicos que as demais espécies, porém seu comportamento foi diferenciado, sendo que todas as frações e extratos não apresentaram diferença significativa entre si.

Resultados semelhantes aos obtidos pelo presente trabalho para o EB do C. annuum var. annuum foram apresentados por Hassimoto, Genovese e Lajolo (2005) para extratos metanólicos de C. annuum var. annuum, utilizando reagente Folin Ciocalteu, com $119 \mathrm{mg} .100 \mathrm{~g}^{-1}$ para pimentas verdes e $131 \mathrm{mg} .100 \mathrm{~g}^{-1}$ para vermelhas.

Deepa et al. (2007) encontraram para extrato etanólico de dez espécies de C. annuum L. concentração de fenólicos totais para pimentas verdes variando de 186 a $1122 \mathrm{mg} .100 \mathrm{~g}^{-1} \mathrm{e}$ para pimentas vermelhas de 323 a $852 \mathrm{mg} / \mathrm{kg}$.

Howard et al. (2000), utilizando o reagente Folin Ciocalteu para extratos metanólicos de pimentas Capsicum, encontraram de 2846 a $5707 \mathrm{mg} / \mathrm{kg}$ de fenólicos totais em frutos maduros de quatro espécies de C. annuum e imaturos de 2565 a $3548 \mathrm{mg} / \mathrm{kg}$; para os frutos imaturos do C. frutescens $5244 \mathrm{mg} / \mathrm{kg}$ e maduros

Tabela 2. Concentração de fenólicos totais $\left(\mathrm{mg} \cdot 100 \mathrm{~g}^{-1}\right)$ de extrato bruto e fracionado de pimentas do gênero Capsicum.

\begin{tabular}{|c|c|c|}
\hline Pimenta & Extrato e frações & Fenólicos totais ${ }^{*}\left(\mathrm{mg} .100 \mathrm{~g}^{-1}\right)$ \\
\hline \multirow[t]{4}{*}{ C. frutescens (malagueta) } & Bruto & $173,19 \pm 6,65^{\mathrm{f}}$ \\
\hline & Hexânico & $173,19 \pm 6,65^{\mathrm{f}}$ \\
\hline & Clorofórmico & $13.311,79 \pm 186,63^{\mathrm{b}}$ \\
\hline & Acetato de etila & $10.725,71 \pm 2632,8^{c}$ \\
\hline Média & & $6.095,97^{\mathrm{B}}$ \\
\hline \multirow[t]{4}{*}{ C. annuum (pimentão magali) } & Bruto & $139,95 \pm 10,16^{\mathrm{f}}$ \\
\hline & Hexânico & $228,60 \pm 29,98^{\mathrm{f}}$ \\
\hline & Clorofórmico & $208,6 \pm 10,16^{\mathrm{f}}$ \\
\hline & Acetato de etila & $135,52 \pm 10,16^{\mathrm{f}}$ \\
\hline Média & & $178,18^{\mathrm{D}}$ \\
\hline \multirow[t]{4}{*}{ C. baccatun var. baccatun (cambuci) } & Bruto & $135,52 \pm 10,16^{\mathrm{f}}$ \\
\hline & Hexânico & $40,21 \pm 6,65^{\mathrm{f}}$ \\
\hline & Clorofórmico & $8.031,88 \pm 645,52^{\mathrm{d}}$ \\
\hline & Acetato de etila & $7.277,61 \pm 493,79^{d}$ \\
\hline Média & & $3.871,30^{\mathrm{C}}$ \\
\hline \multirow[t]{4}{*}{ C.baccatun var. praetermissum (cumari } & Bruto & $177,63 \pm 7,68^{\mathrm{f}}$ \\
\hline & Hexânico & $126,65 \pm 6,65^{\mathrm{f}}$ \\
\hline & Clorofórmico & $16.867,65 \pm 186,63^{\mathrm{a}}$ \\
\hline & Acetato de etila & $16.975,41 \pm 186,63^{a}$ \\
\hline Média & & $8.536,83^{\mathrm{A}}$ \\
\hline
\end{tabular}

\footnotetext{
${ }^{*}$ Dados expressos como miligramas equivalentes de catecol por $100 \mathrm{~g}$ de extrato.
} 
$5136 \mathrm{mg} / \mathrm{kg}$; e para a espécie C. chinense frutos maduros $4042 \mathrm{mg} / \mathrm{kg}$ de fenólicos totais. Demonstrando a correlação positiva entre o aumento da maturidade com a concentração de fenólicos para a maioria das pimentas testadas. O contrário foi relatado por Marin et al. (2004): houve decréscimo do conteúdo de fenólicos com o estágio de maturação do verde para vermelho.

\subsection{Avaliação da atividade antioxidante pelo sistema $\beta$-caroteno ácido linoleico}

O sistema $\beta$-caroteno/ácido linoleico baseia-se na descoloração do $\beta$-caroteno induzida pelos produtos da oxidação do ácido linoleico. A utilização de antioxidantes retarda a queda da absorbância do $\beta$-caroteno, protegendo os substratos lipídicos da oxidação (SOKMEN et al., 2004).

Os resultados da atividade antioxidante (\%) pelo Sistema $\beta$-caroteno Ácido Linoleico, dos extratos bruto e fracionado da pimenta malagueta (C.frutescens), pimentão magali (C. annuum var. annuum), pimenta cambuci (C.baccatum var. pendulum) e pimenta cumari (C.Baccatum var. praetermissum), encontramse na Tabela 3.

Observou-se que o EB da malagueta (C. frutescens) apresentou a maior $(\mathrm{p}<0,05)$ atividade antioxidante e a FA a menor ( $\mathrm{p}<0,05)$, ficando a FC e FH com valores intermediários. Comparando-se com o antioxidante sintético (BHT), o EB, FH e FC da malagueta obtiveram a mesma ação antioxidante, e a FA com atividade inferior $(\mathrm{p}<0,05)$.
Em virtude de a pimenta malagueta ter apresentado maior concentração de fenólicos nas frações clorofórmica e acetato de etila e menores na hexânica e no extrato bruto, esperava-se que as frações com maiores concentrações de fenólicos apresentassem maior atividade antioxidante. Porém, observou-se o contrário, principalmente com a fração acetato que apresentou menor atividade antioxidante. Este resultado possivelmente deveu-se à presença da vitamina $\mathrm{C}$ extraída nesta fração, que agiu como pró-oxidante, uma vez que, segundo Markus et al. (1999) e Reifschneider (2000), este gênero se apresenta como boa fonte deste nutriente. Conforme Hassimoto, Genovese e Lajolo (2005), o ácido ascórbico pode atuar como pró-oxidante, pois ao doar os dois hidrogênios redutores, fica susceptível de receber elétrons, devido ao radical ascorbila formado, que é agente oxidante.

A baixa atividade antioxidante do extrato bruto e fração hexânica da pimenta malagueta deveu-se principalmente à ação sinergística entre os compostos presentes (EB) e a presença de capsantinas e criptoxantina (FH), que são carotenoides com ação antioxidante. Segundo Young e Lowe (2001), os carotenoides apresentam propriedades antioxidantes devido principalmente ao sistema de duplas ligações conjugadas, fazendo com que sejam capazes de capturar os radicais livres.

Howard et al. (2000) obtiveram para o extrato metanólico da pimenta tabasco (C. frutescens), fruto maduro, 91,85\% de atividade inibitória da oxidação pelo sistema $\beta$-caroteno/ Ácido Linoleico, e correlacionaram o efeito antioxidante com as concentrações crescentes de carotenoides nos frutos maduros: $414 \mu \mathrm{g} .100 \mathrm{~g}^{-1}$ de $\beta$-criptoxantina, $1252 \mu \mathrm{g} .100 \mathrm{~g}^{-1}$

Tabela 3. Atividades antioxidantes (\%) dos extratos bruto e fracionados de pimentas Capsicum e BHT, pelo sistema â $\beta$-caroteno/ácido linoleio.

\begin{tabular}{|c|c|c|}
\hline Pimentas & Extrato e frações & Atividade antioxidante $\%$ \\
\hline \multirow[t]{4}{*}{ C. frutescens (Malagueta) } & EB & $87,80 \pm 1,21^{\mathrm{ab}}$ \\
\hline & $\mathrm{FH}$ & $85,07 \pm 1,15^{\mathrm{bc}}$ \\
\hline & $\mathrm{FC}$ & $82,63 \pm 1,63^{\mathrm{bc}}$ \\
\hline & FA & ${ }^{\star} 62,53 \pm 7,52^{\mathrm{d}}$ \\
\hline Média & & $79,53^{\text {B }}$ \\
\hline \multirow[t]{4}{*}{ C. annuum var. annuum (pimentão magali) } & $\mathrm{EB}$ & $\star 33,76 \pm 5,69^{f}$ \\
\hline & $\mathrm{FH}$ & ${ }^{*} 47,82 \pm 2,42^{\mathrm{e}}$ \\
\hline & FC & $\star 35,54 \pm 2,68^{\mathrm{f}}$ \\
\hline & FA & $* 30,80 \pm 5,53^{\mathrm{f}}$ \\
\hline Média & & $36,98^{\mathrm{C}}$ \\
\hline \multirow[t]{4}{*}{ C. baccatun var. pendulum (cambuci) } & $\mathrm{EB}$ & $96,97 \pm 3,03^{\mathrm{a}}$ \\
\hline & $\mathrm{FH}$ & $\star 77,78 \pm 1,75^{\mathrm{bc}}$ \\
\hline & FC & ${ }^{\star} 78,79 \pm 3,03^{\mathrm{bc}}$ \\
\hline & FA & $96,97 \pm 0^{\mathrm{a}}$ \\
\hline Média & & $87,63^{\mathrm{A}}$ \\
\hline \multirow[t]{4}{*}{ C. baccatun var. praetermissum (cumari) } & $\mathrm{EB}$ & ${ }^{*} 75,76 \pm 3,03^{c}$ \\
\hline & $\mathrm{FH}$ & ${ }^{\star} 62,63 \pm 6,31^{\mathrm{d}}$ \\
\hline & FC & $81,81 \pm 0^{\mathrm{bc}}$ \\
\hline & FA & $86,87 \pm 4,63^{\mathrm{abc}}$ \\
\hline Média & & $76,77^{\mathrm{B}}$ \\
\hline Aditivo sintético & BHT & $88,65 \pm 1,23$ \\
\hline
\end{tabular}

Médias seguidas pelas mesmas letras minúsculas (média dos extratos) e maiúsculas (média das pimentas) na coluna, não diferem (p >0,05) pelo teste Tukey. ${ }^{\star}$ Médias diferentes ( $>$ ou $<$ ) em relação ao antioxidante sintético (BHT), pelo teste Dunnett a 5\%. 
de $\alpha$-caroteno, $1187 \mu \mathrm{g} .100 \mathrm{~g}^{-1}$ de $\beta$-caroteno, $1443 \mu \mathrm{g} .100 \mathrm{~g}^{-1}$ de capsantina e $1958 \mu \mathrm{g} .100 \mathrm{~g}^{-1}$ de zeaxantina.

Para o pimentão magali (C. annuum), a maior atividade antioxidante foi obtida pela $\mathrm{FH}$, sendo os menores valores atribuídos ao EB, FC eFA, que não diferenciaram estatisticamente entre si. $\mathrm{O}$ extrato bruto e todas as frações apresentaram valores inferiores ao BHT.

Observou-se que o EB e FA da pimenta cambuci (C. baccatum var. pendulum) apresentaram maior ação antioxidante e igual ao BHT, ficando a FH e FC com menor atividade e inferiores ao BHT.

A ação antioxidante do extrato em acetato de etila da pimenta cambuci justifica-se pela presença significativa de capsaicinoides e fenólicos totais encontrados nesta fração. Apesar de a fração clorofórmica ter apresentado a mesma concentração de capsaicinoides e fenólicos totais que a fração acetato de etila, sua ação antioxidante possivelmente foi afetada por outros compostos que interagiram de forma negativa neste sistema.

Para a pimenta cumari (C. baccatun var. praetermissum), as frações acetato de etila e clorofórmica obtiveram os melhores resultados com valores iguais ao $\mathrm{BHT}$, ficando a $\mathrm{FH}$ com a menor ação antioxidante e o EB com a atividade intermediária, ambos com valores inferiores ao BHT.

Os resultados apresentados pelas frações acetato de etila e clorofórmica da pimenta cumari podem ser explicados pela alta concentração de capsaicinoides e fenólicos totais encontrados nestas frações. Os valores do EB e FH podem ser explicados pela pouca quantidade de compostos como os carotenoides, além das baixas concentrações de capsaicinoides e fenólicos totais detectados neste extrato e fração.

A pimenta cambuci apresentou a maior $(\mathrm{p}<0,05)$ ação antioxidante, seguida da pimenta malagueta e cumari, sendo a menor $(\mathrm{p}<0,05)$ atividade obtida pelo pimentão.

Observou-se que a ação antioxidante do EB foi maior que a fração acetato de etila, com menor ação nas frações bichano e clorofórmio. A melhor atividade foi obtida pela FA e EB da pimenta cambuci, enquanto que o EB e todas as frações do pimentão atingiram a menor ação antioxidante. Isso porque os resultados dependem, segundo Dorman et al. (2003), da natureza e estrutura química dos compostos fenólicos presentes no extrato. Também os compostos que possuem ação antioxidante podem variar em função da espécie de pimenta, das suas condições de cultivo e da forma de extração, afetando diretamente a atividade inibitória do extrato (DEANS; RITCHIE, 1987).

Utilizando-se como parâmetro a classificação de Hassimotto, Genovese e Lajolo (2005), em que valores de inibição > 70\% apresentaram boa ação, intermediária para valores de 40 a 70\% de inibição e baixa para < $40 \%$ de inibição, observou-se que: os extratos e frações das pimentas malagueta, cumari e cambuci apresentaram boa ação antioxidante, sendo apenas o pimentão com valores menores classificado como de baixa atividade antioxidante.
Hassimotto, Genovese e Lajolo (2005), ao avaliar a atividade antioxidante C. annuum var. annuum na concentração de $50 \mu \mathrm{M}$ de extrato metanólico pelo Sistema $\beta$-caroteno/Ácido Linoleico, também obtiveram baixa ( $<40 \%$ inibição) ação antioxidante para pimenta doce verde, $31,4 \%$ de inibição da oxidação, enquanto que para pimenta doce vermelha, uma atividade de 49,2\% de inibição, considerada atividade intermediária (40-70\% de inibição).

\subsection{Avaliação da atividade antioxidante pelo método DPPH (2,2-difenil-1-picrilhidrazila)}

O método DPPH consiste na redução do radical DPPH. (2,2-difenil-1-piricrilhidrazila), de coloração púrpura, que, ao receber um elétron ou um radical hidrogênio, muda sua coloração de violeta para amarelo (difenil-piricril-hidrazina), ficando estável e com o desaparecimento da absorção que pode ser avaliada pelo decréscimo da absorbância (ROGINSKY; LISSI, 2005).

Encontram-se na Tabela 4 os resultados da atividade antioxidante (\%) pelo método DPPH, dos extratos bruto e fracionado da pimenta malagueta (C. frutescens), pimentão magali (C. annuum var. annuum), pimenta cambuci (C.baccatum var pendulum.) e pimenta cumari (C.Baccatum var. praetermissum).

Observa-se que as FA e FC da pimenta malagueta (C. frutescens) apresentaram maior $(\mathrm{p}<0,05)$ atividade antioxidante em todas as concentrações testadas, com desempenho igual a superior a $\mathrm{BHT}$. Tais resultados podem ser explicados pela considerável concentração de fenólicos totais e capsaicinoides encontrados nestas frações, os quais possuem grande capacidade de doar elétrons ao radical DPPH e estabilizá-lo. Enquanto que o EB obteve menor ação do que o $\mathrm{BHT}$, provavelmente devido à interação negativa de compostos variados extraídos pelo etanol, ou pela presença de substâncias com menor poder redutor.

A atividade antioxidante do pimentão magali (C. annuum var. annuum) em média apresentou ação inferior ao BHT, ficando a fração clorofórmica apenas nas concentrações de 50 e $250 \mu \mathrm{g} \cdot \mathrm{mL}^{-1}$ com ação igual ao antioxidante sintético, e a fração acetato de etila com desempenho superior ao BHT apenas nas concentrações de 125 e $250 \mu \mathrm{g} \cdot \mathrm{mL}^{-1}$. Isto demonstra que somente em altas concentrações as frações clorofórmica e acetato de etila foram eficientes doadores de elétrons para o radical DPPH. Enquanto que o extrato bruto obteve menor ação antioxidante mesmo apresentando concentrações de fenólicos e capsaicinoides iguais aos extratos brutos das outras pimentas estudadas.

Materska e Perucka (2005), ao avaliarem a atividade antioxidante da capsaicina e dihidrocapsaicina extraídas do C. annuum L., verificaram pelo método DPPH que a ação antioxidante da capsaicina foi maior que a da dihidrocapsaicina, mostrando que a dupla ligação da cadeia lipídica da capsaicina influenciou na ação antioxidante. Acrescentaram que a ação dos capsaicinoides pode ter sido influenciada pela presença dos grupos metoxi e $\mathrm{OH}$ na posição orto do anel aromático. 
Costa et al.

Tabela 4. Atividades antioxidantes (\%) obtidos em diversas concentrações dos extratos bruto e fracionados das pimentas Capsicum e BHT, pelo método DPPH.

\begin{tabular}{|c|c|c|c|c|c|c|c|}
\hline \multirow[t]{2}{*}{ Pimentas } & \multirow{2}{*}{$\begin{array}{c}\text { Extrato/ } \\
\text { frações }\end{array}$} & \multicolumn{6}{|c|}{ Concentração de extrato/frações $\left(\mu \mathrm{g} \cdot \mathrm{mL}^{-1}\right)$} \\
\hline & & 5 & 10 & 25 & 50 & 125 & 250 \\
\hline \multirow[t]{3}{*}{ C. frutescens (malagueta) } & EB & ${ }^{\star} 13,90 \pm 3,3^{\text {cd }}$ & ${ }^{\star} 16,08 \pm 3,05^{\mathrm{de}}$ & $\star 20,13 \pm 5,3^{\text {de }}$ & $\star 23,34 \pm 2,62^{\mathrm{de}}$ & ${ }^{*} 46,01 \pm 13,4^{\mathrm{de}}$ & $\star 56,91 \pm 9,91^{c}$ \\
\hline & $\mathrm{FC}$ & ${ }^{\star} 40,69 \pm 5,60^{\mathrm{a}}$ & $40,71 \pm 9,06^{\mathrm{ab}}$ & $55,76 \pm 7,48^{\mathrm{c}}$ & $\star 55,76 \pm 1,56^{\mathrm{de}}$ & ${ }^{*} 85,95 \pm 1,19^{\mathrm{ab}}$ & $86,41 \pm 1,22^{\mathrm{ab}}$ \\
\hline & FA & $17,44 \pm 3,15^{c}$ & $21,71 \pm 3,57^{\mathrm{cd}}$ & $39,95 \pm 3,36^{\mathrm{c}}$ & $* 64,81 \pm 1,09^{\mathrm{cd}}$ & ${ }^{*} 92,15 \pm 1,92^{\mathrm{a}}$ & $\star 95,90 \pm 2,97^{\mathrm{a}}$ \\
\hline Média & & $24,01^{\mathrm{A}}$ & $26,17^{\mathrm{A}}$ & $34,64^{\mathrm{A}}$ & $47,97^{\mathrm{C}}$ & $74,70^{\mathrm{A}}$ & $79,74^{\mathrm{AB}}$ \\
\hline \multirow{3}{*}{$\begin{array}{l}\text { C. annuum var. annuum } \\
\text { (Pimentão) }\end{array}$} & $\mathrm{EB}$ & $17,29 \pm 1,32^{\mathrm{c}}$ & ${ }^{\star} 17,57 \pm 1,45^{\mathrm{de}}$ & $22,47 \pm 6,44^{\mathrm{d}}$ & $\star 23,51 \pm 6,43^{\mathrm{g}}$ & ${ }^{\star} 41,88 \pm 8,52^{\mathrm{e}}$ & $\star 58,40 \pm 2,03^{c}$ \\
\hline & FC & $\star 5,48 \pm 2,32^{\mathrm{de}}$ & ${ }^{*} 10,59 \pm 9,19^{\text {def }}$ & ${ }^{*} 20,46 \pm 1,32^{\mathrm{de}}$ & $35,35 \pm 1,09^{\mathrm{f}}$ & $57,34 \pm 1,32^{\mathrm{d}}$ & $84,24 \pm 1,74^{\mathrm{ab}}$ \\
\hline & FA & 0 & 0 & $\star 9,43 \pm 3,32^{\mathrm{e}}$ & $46,46 \pm 7,38^{\mathrm{e}}$ & ${ }^{*} 90,68 \pm 3,31^{\mathrm{bc}}$ & ${ }^{\star} 96,40 \pm 1,08^{\mathrm{a}}$ \\
\hline Média & & $7,59^{\mathrm{C}}$ & $9,39^{\text {B }}$ & $17,45^{\text {в }}$ & $53,93^{\mathrm{D}}$ & $63,30^{\text {B }}$ & $79,68^{\text {в }}$ \\
\hline \multirow{3}{*}{$\begin{array}{l}\text { C. baccatum var. pendulum } \\
\text { (cambuci) }\end{array}$} & $\mathrm{EB}$ & $* 3,09 \pm 0,29^{\mathrm{e}}$ & $* 4,51 \pm 1,17^{\mathrm{ef}}$ & $10,63 \pm 1,24^{\mathrm{de}}$ & $\star 22,67 \pm 3,21^{\mathrm{g}}$ & $\star 45,57 \pm 2,01^{\mathrm{de}}$ & $82,00 \pm 2,18^{\mathrm{ab}}$ \\
\hline & FC & ${ }^{\star} 13,98 \pm 3,64^{\mathrm{c}}$ & ${ }^{\star} 19,33 \pm 1,31^{\mathrm{d}}$ & $36,08 \pm 8,07^{\mathfrak{c}}$ & $53,47 \pm 2,57^{e}$ & $* 76,78 \pm 4,5^{\mathbf{b}}$ & $88,89 \pm 1,77^{\mathrm{ab}}$ \\
\hline & FA & $28,59 \pm 2,13^{b}$ & $\star 52,31 \pm 5,10^{\mathrm{a}}$ & ${ }^{\star} 85,27 \pm 0,94^{\mathrm{a}}$ & ${ }^{\star} 85,64 \pm 0,78^{a}$ & ${ }^{*} 87,52 \pm 0,94^{\mathrm{ab}}$ & $\star 90,51 \pm 1,2^{\mathrm{a}}$ \\
\hline Média & & $15,22^{\mathrm{B}}$ & $25,38^{\mathrm{A}}$ & $43,99^{\mathrm{A}}$ & $53,93^{\text {В }}$ & $69,96^{\mathrm{A}}$ & $87,71^{\mathrm{A}}$ \\
\hline \multirow{3}{*}{$\begin{array}{l}\text { C. baccatum var. } \\
\text { praetermissum (cumari) }\end{array}$} & $\mathrm{EB}$ & ${ }^{\star} 1,09 \pm 019^{\mathrm{e}}$ & ${ }^{\star} 5,13 \pm 2,48^{\mathrm{ef}}$ & ${ }^{*} 12,25 \pm 0,98^{\mathrm{de}}$ & ${ }^{*} 28,63 \pm 3,80^{\mathrm{fg}}$ & $54,42 \pm 2,15^{\mathrm{de}}$ & $73,22 \pm 16,04^{b c}$ \\
\hline & FC & ${ }^{\star} 31,55 \pm 2,87^{\mathbf{b}}$ & $40,75 \pm 2,00^{\mathrm{ab}}$ & ${ }^{*} 59,54 \pm 2,32^{\mathrm{b}}$ & ${ }^{\star} 74,81 \pm 1,75^{\mathrm{bc}}$ & ${ }^{*} 83,63 \pm 0,73^{\mathrm{ab}}$ & $89,57 \pm 1,17^{\mathrm{ab}}$ \\
\hline & FA & $18,83 \pm 2,45^{\mathrm{c}}$ & $34,48 \pm 3,61^{\mathrm{bc}}$ & $* 57,25 \pm 1,99^{\mathrm{b}}$ & $\star 80,03 \pm 1,10^{\mathrm{ab}}$ & ${ }^{*} 90,46 \pm 0,77^{\mathrm{ab}}$ & ${ }^{*} 91,35 \pm 1,96^{\mathrm{a}}$ \\
\hline Média & & $17,16^{\mathrm{B}}$ & $26,79^{\mathrm{A}}$ & $43,01^{\mathrm{A}}$ & $61,16^{\mathrm{A}}$ & $76,17^{\mathrm{A}}$ & $84,71^{\mathrm{AB}}$ \\
\hline Sintético & BHT & $21,76 \pm 3,4$ & $31,52 \pm 1,17$ & $32,90 \pm 6,8$ & $43,58 \pm 12,5$ & $60,1 \pm 5,03$ & $77,02 \pm 0,97$ \\
\hline
\end{tabular}

Médias seguidas pelas mesmas letras minúsculas (média dos extratos) e maiúsculas (média das pimentas) na coluna, não diferem ( $\mathrm{p}>0,05)$ pelo teste Tukey. ${ }^{*}$ Médias diferentes $(>$ ou $<$ ) em relação ao antioxidante sintético (BHT), pelo teste Dunnett a $5 \%$.

Observa-se que a fração acetato de etila da pimenta cambuci (C. baccatum var baccatum) ofereceu maior contribuição para atividade antioxidante desta espécie, com capacidade doadora de elétron superior ao antioxidante sintético BHT. Enquanto que a fração clorofórmica obteve valores variando entre ação antioxidante inferior a igual ao BHT, o extrato bruto apresentou atividade inferior ao BHT.

A concentração de fenólicos totais encontrada na fração acetato de etila da pimenta cambuci justifica a ação antioxidante superior ao BHT, já que apresentou concentração baixa de capsaicinoides. A fração clorofórmica apesar de ter apresentado concentração de fenólicos totais igual ao extrato de acetato de etila, sua ação antioxidante pode ter sido afetada por outros compostos presentes nesta fração. As concentrações inferiores de capsaicinoides e fenólicos totais no extrato bruto explicam sua menor atividade antioxidante quando comparadas com as frações.

Os compostos contidos nas frações clorofórmica e acetato de etila da pimenta cumari (C. baccatum var. praetermissum) atingiram ação antioxidante superior ao $\mathrm{BHT}$, o que provavelmente deveu-se à elevada concentração de capsaicinoides e fenólicos totais detectada nestas frações. Já o extrato bruto obteve uma ação antioxidante igual ao BHT apenas nas concentrações de 125 e $250 \mu \mathrm{g} \cdot \mathrm{mL}^{-1}$.

A atividade antioxidante das frações hexânicas não foi determinada, pois os resultados se apresentavam abaixo de zero, impossibilitando o cálculo da atividade e o cálculo do $\mathrm{CE}_{50}$ através de regressão linear, podendo ser explicado devido à baixa concentração de fenólicos totais encontrada para esta fração. Segundo Wangensteen, Samuelsen e Malterrud (2004), a falta de capacidade doadora de hidrogênio dos extratos lipofílicos dificulta a análise de seus resultados pela metodologia do DPPH.

Observou-se na Figura 1 que os valores de $\mathrm{CE}_{50}$ foram menores para as frações acetato de etila e clorofórmica e semelhantes ao BHT, porém maiores para os extratos brutos. O que é justificado pela maior concentração de capsaicinoides e fenólicos totais encontrados nestas frações polares.

A pimenta cumari foi a pimenta que apresentou os menores $(\mathrm{p}<0,05)$ valores de $\mathrm{CE}_{50}$ quando comparados aos das outras pimentas e BHT. Para o pimentão, o extrato bruto e suas frações atingiram os maiores valores de $\mathrm{CE}_{50}$. A pimenta malagueta apresentou altos valores de $\mathrm{CE}_{50}$ para o extrato bruto e suas frações com resultados semelhantes aos da cambuci.

Os extratos da pimenta cumari apresentaram a maior $(\mathrm{p}<0,05)$ ação doadora de elétrons ao radical DPPH, seguida da pimenta cambuci, malagueta e pimentão. Os extratos clorofórmico e acetato de etila obtiveram a melhor atividade antioxidante, quando comparados ao BHT e ao extrato bruto. Demonstrando correlação positiva entre ação antioxidante e as concentrações de capsaicinoides e fenólicos totais encontrados nas frações polares (acetato de etila e clorofórmico).

Observou-se que o FA apresentou resultado contrário ao que foi obtido com o Sistema $\beta$ - caroteno/Ácido Linoleico para pimenta malagueta e pimentão, isto porque o método DPPH, segundo Duarte-Almeida et al. (2006), avalia apenas o poder redutor do antioxidante, que ao doar elétron se oxida e, por isso, não detecta substâncias pró-oxidantes como ácido ascórbico. Enquanto que o Sistema $\beta$-caroteno/Ácido Linoleico avalia 


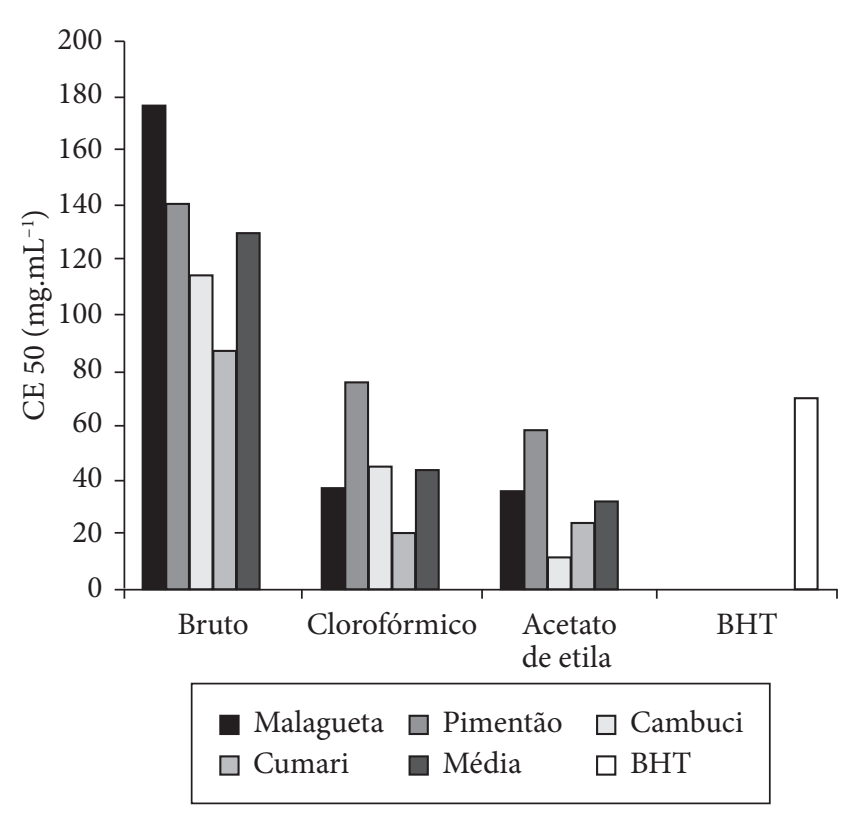

Figura 1. Atividade antioxidante $\left(\mathrm{CE}_{50}\right)$ do extrato bruto e fracionado de pimentas Capsicum, pelo método DPPH.

a capacidade que o antioxidante possui em proteger o ácido Linoleico e o $\beta$-caroteno da oxidação, identificando substâncias pró-oxidantes como a vitamina $\mathrm{C}$.

Constatou-se que a metodologia do radical DPPH detectou muito mais a ação antioxidante dos extratos polares, que extraem os compostos fenólicos, do que o extrato bruto. Conforme Atoui et al. (2005), a atividade antioxidante dos fenólicos é devida principalmente às suas propriedades redutoras.

\section{Conclusões}

O C. baccatun var. praetermissum (cumari), o C. baccatun var. pendulum (cambuci) e o C. frutescens (malagueta) podem ser utilizados como agentes antioxidantes naturais em alimentos. Já o C. annuum var. annuum (pimentão magali) apresentou bom desempenho apenas em concentrações elevadas das frações polares pelo método DPPH.

\section{Referências bibliográficas}

ASSOCIATION OF OFFICIAL ANALYTICAL CHEMISTS - AOAC. Official methods of analysis. 14. ed. Washington, D.C., 1980.

ATOUI, A. K. et al. Tea and herbal infusions: their antioxidant activity and phenolic profile. Food Chemistry, v. 89, n. 1, p. 27-36, 2005.

BERTOLDI, M. C. Atividade antioxidante in vitro da fração fenólica, das oleorresinas e do óleo essencial de pimenta rosa (Schinus terebinthifolius Raddi). 2006, 96 p. Dissertação (Mestrado em Ciência e Tecnologia de Alimentos) - Universidade Federal de Viçosa - UFV, Viçosa, MG, 2006.

BRAND-WILLIAMS, W.; CUVELIER, M. E.; BERSET, C. Use of free radical method to evaluate antioxidant activity. LebensmittelWissenschaft und-Technologie, v. 28, p. 25-30, 1995.
CARVALHO, S. I. C.; BIANCHETTI, L. B. Sistema de produção de pimentas. 2004. Disponível em: <http://www.cnph.embrapa.br/ sistprod/pimenta/botanica.htm>. Acesso em: 01 fev. 2006.

DEANS, S. G.; RITCHIE, G. Antibacterial properties of plant essencial oils. International Journal of Food Microbiology, v. 5, p. 165-180, 1987.

DEEPA, N. Antioxidant constituents in some sweet pepper (Capsicum annuиm L.) genotypes during maturity. LWT - Food Science and Technology, v. 40, p. 121-129, 2007.

DORMAN, H. J. D. Characterisation of the antioxidant properties of de-odourised aqueous extracts from selected mamiaceae herbs. Food Chemistry, v. 83, n. 2, p. 255-262, 2003.

DUARTE-ALMEIDA, J. M. Avaliação da atividade antioxidante utilizando sistema $\beta$-caroteno/ácido Linoléico e método de seqüestro de radicais livres. Ciência e Tecnologia de Alimentos, v. 26, n. 2, p. 446-452, 2006.

HASsimotTo, N. M. A.; GENOVESE, M. I.; LAJOlO, F. M. Antioxidant activity of dietary fruits, vegetables, and commercial frozen fruit pulps. Journal of Agricultural and Food Chemistry, v. 53, p. 2928-2935, 2005.

HOWARD, L. R. Changes in phytochemical and antioxidant activity of selected pepper cultivars (Capsicum Species) as influenced by maturity. Journal of Agricultural and Food Chemistry, v. 48, p. 1713-1720, 2000.

MARCO, G. J. A rapid method for evolution of antioxidants. Journal of the Americam Oil Chemists' Society, v. 45, p. 594-598, 1968.

MARIN, A. et al. Characterization and quantification of antioxidant constituents of sweet (Capsicum annuum L.). Journal of Agricultural and Food Chemistry, v. 52, p. 3861-3869, 2004.

MARKUS, F. et al. Change in the carotenoid and antioxidant content of spice red pepper (paprika) as a function of ripening and some techonological factors. Journal of Agricultural and Food Chemistry, v. 47, p. 100-107, 1999.

MATERSKA, M.; PERUCKA, I. Antioxidant activity of the main phenolic compounds isolated from hot pepper fruit (Capsicum annuum L.). Journal of Agricultural and Food Chemistry, v. 53, p. 1750-1756, 2005.

MENSOR, L. L. Screening of Brazilian plant extracts for antioxidant activity by the use of DPPH free radical method. Phytoterapy Research, v. 15, p. 127-130, 2001.

PERUCKA, I.; OLESZEK, W. Extraction and determination of capsaicinoids in fruit of hot pepper Capsicum annuum L. by spectrophotometry and high-performance liquid chromatography. Food Chemistry, v. 71, p. 287-291, 2000.

PINO, J. et al. Characterization of total capsaicinoids, colour and volatile compounds of Habanero chilli pepper (Capsicum chinense Jack) cultivars grown in Yucatan. Food Chemistry, v. 104, n. 4, p. 1682-1686, 2007.

REIFSCHNEIDER, F. J. B. (Org.) Capsicum: pimentas e pimentões no Brasil. Brasília: Embrapa Comunicação para Transferência de Tecnologia/Embrapa Hortaliças, 2000.

RISTORI, C. A.; PEREIRA, M. A. S.; GELLI, D. S. O efeito da pimenta do reino moída frente a contaminação in vitro com Salminella Rubslaw. Rev. Inst. Adolfo Lutz, v. 62, n. 2, p. 131-133, 2002.

ROGINSKY, V.; LISSI, E. A. Review of methods to determine chainbreaking antioxidant activity in food. Food Chemistry, v. 92, p. 235-254, 2005.

SADASIVAM, S.; MANIKKAM, A. Capsaicin. In: Biochamical methods for agricultural sciences. New Delhi: Wiley Eastern Limited, 1992. p. 193-194. 
SOKMEN, A. et al. The in vitro antimicrobial and antioxidant activies of the essencial oils and methanol extracts of endemic Thymus spathulifolius. Food Chemistry, n. 15, p. 627-634, 2004.

TOPUZ, A.; OZDEMIR, F. Assessment of carotenoids and ascorbic acid compositiom of some selected pepper cultivars (Capsicum annuum L.) grown in Turkey. Journal of Food Composition and Analysis, v. 20, n. 7, p. 596-602, 2007.

WAGNER, C. M. Variedade e base genética da pungência e da caracteres do fruto: implicações no melhoramento de uma população de Capsicum annunnm L. 2003. 104 p. Dissertação
(Mestrado Genética e Melhoramento de Plantas) - Escola Superior de Agricultura "Luiz de Queiroz", Universidade de São Paulo, Piracicaba, 2003.

WANGENSTEEN, H.; SAMUELSEN, A. B.; MALTERRUD, K. E. Antioxidant activity in extracts from coriander. Food Chemistry, v. 88 , p. $293-297,2004$.

YOUNG, A.; LOWE, G. M. Antioxidant and prooxidant proprieties of carotenoids. Archives of Biochemistry and Biophysics, v. 385, p. 20-27, 2001. 